\title{
SORVEGLIANZA DELLE INFEZIONI \\ OSPEDALIERE: \\ OSSERVAZIONI PRESSO IL PRESIDIO \\ OSPEDALIERO DI PENNE (PE).
}

\author{
Ridolfi D., Della Pelle C., Savini F.,Tresca E. \\ Laboratorio Analisi Chimico Cliniche e Microbiologia, AUSL \\ Pescara, P.O. "S. Massimo", Penne(PE).
}

Il Laboratorio di Microbiologia rappresenta un'utile fonte informativa per il controllo delle infezioni ospedaliere in quanto consente di identificare eventi sentinella, epidemie, presenza di microrganismi multiresistenti e monitorare l'andamento delle resistenze stesse.

Scopo del lavoro: valutare la frequenza di isolamento delle varie specie batteriche nel complesso dei pazienti ospedalizzati, la sensibilità agli antibiotici e valutare la diffusione dello Staphylococcus aureus meticillino-resistente(MRSA) tra gli Operatori Sanitari.

Materiali e metodi: sono stati considerati tutti i ceppi batterici isolati da materiali biologici (urine,sangue,punte di catetere, espettorati, liquidi pleurici, lavaggi bronchiali,tamponi da ferita,pus).Gli stipiti batterici sono stati identificati e gli antibiogrammi eseguiti utilizzando il sistema Vitek (BioMerieux). Sono stati effettuati i tamponi nasali a 92 Operatori Sanitari dei reparti chirurgici, delle sale operatorie e rianimazione per ricercare lo Staphylococcus aureus meticillino-resistente.

Risultati: nei pazienti ricoverati dal 01/01/2000-31/12/2004 sono stati isolati 1754 microrganismi. I batteri di più frequente riscontro sono stati nell'ordine: Escherichia coli (38\%), Staphylococcus aureus (10.6\%), Enterococcus faecalis $(9.7 \%)$, Pseudomonas aeruginosa $(8.7 \%)$, Proteus mirabilis $(8.5 \%)$, Klebsiella pneumoniae (6.5\%), Staphylococcus 
coagulasi negativo (5.7\%),Enterobacter spp. (5.6\%). Gli Stafilococchi meticillino-resistenti, gli Pseudomonas multiresistenti e gli Enterobacter si confermano come importanti patogeni nosocomiali.

Nei ceppi di Staphylococcus aureus isolati, la resistenza all'oxacillina è stata del $32 \%$ e nei Staphylococcus coagulasi negativi del $71 \%$. Tra le Enterobatteriaceae la specie più resistente si è dimostrata Enterobacter spp.

Nei ceppi di Pseudomonas aeruginosa, l'imipenem ha presentato in vitro la migliore attività antimicrobica (sensibilità $82 \%)$.

Il $40 \%$ del personale è colonizzato da Staphylococcus aureus ma nessuno è risultato portatore di MRSA.

Conclusioni: la sorveglianza continuativa mediante il Laboratorio di Microbiologia rende più facile la predisposizione di piani di controllo delle infezioni ospedaliere. 